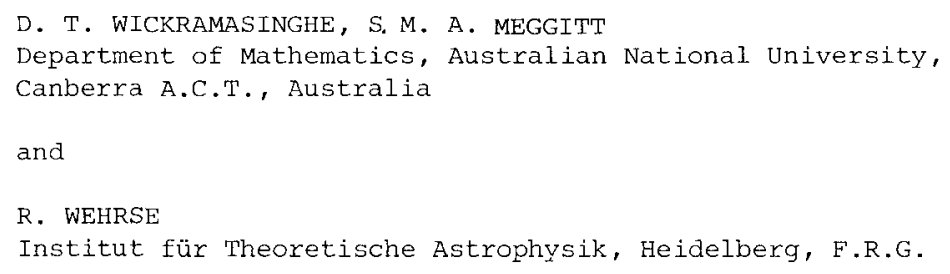

\begin{abstract}
The intensity and polarisation properties of magnetic accretion columns are discussed in the regime where electron scattering is important. It is shown that the circular polarisation standstills observed in some AM Herculis type systems are inconsistent with cyclotron emission from scattering dominated regions.
\end{abstract}

\title{
Introduction
}

The emission properties of accretion shocks giving rise to the polarised light observed in AM Herculis type systems have been discussed by various investigators (Meggitt and Wickramasinghe (1982), Chanmugam and Dulk (1981), Wickramasinghe and Meggitt (1985a)). Most studies have neglected the effects of electron scattering within the cyclotron emission region. The possible importance of scattering was first noted by Barrett and Chanmugam (1984) who constructed detailed low temperature scattering dominated models for AM Herculis assuming a plane parallel geometry for the emission region. Their 
calculations were carried out under the assumption that scattering could be included as extinction and thermal emission terms in the transfer equations. Wickramasinghe and Meggitt (1985a) have noted that this assumption is likely to lead to erroneous results under the conditions expected in AM Herculis type systems. In this paper we present results of calculations of magnetic accretion columns which include the effects of scattering in a more realistic manner and discuss these in relation to the observed properties of AM Herculis type systems.

\section{Calculations and Discussion}

In the limit of large Faraday rotation the polarised transfer problem can be formulated in terms of the intensities $I_{1}$ and $I_{2}$ of the normal modes of propagation. When electron scattering is important the equations take the form (Kaminker, Pavlov and Shibanov (1982))

$$
\begin{aligned}
& \frac{d I_{1}(\underline{n})}{d s}=-\kappa_{1}^{a}(\underline{n})\left[I_{1}(\underline{n})-B\right]-\sigma_{1}(\underline{n}) I_{1}(\underline{n}) \\
& +\iint\left[\sigma_{11}\left(\underline{\mathbf{n}}, \underline{\underline{n}}^{\prime}\right) \mathrm{I}_{1}\left(\underline{\underline{\prime}}^{\prime}\right)+\sigma_{12}\left(\underline{\mathrm{n}}, \underline{\underline{n}}^{\prime}\right) \mathrm{I}_{2}\left(\underline{\underline{n}}^{\prime}\right) \mathrm{d} \omega\left(\underline{\underline{n}}^{\prime}\right)\right. \\
& \frac{d I_{2}(\underline{n})}{d s}=-\kappa_{2}^{a}(\underline{n})\left[I_{2}(\underline{n})-B\right]-\sigma_{2}(\underline{n}) I_{2}(\underline{n}) \\
& +\iint\left[\sigma_{21}\left(\underline{n}, \underline{n}^{\prime}\right) I_{1}\left(\underline{n}^{\prime}\right)+\sigma_{22}\left(\underline{n}, \underline{n}^{\prime}\right) I_{2}\left(\underline{n}^{\prime}\right)\right] d \omega\left(\underline{n}^{\prime}\right)
\end{aligned}
$$

where ds is the element of length in direction $\underline{\mathbf{n}} \cdot \sigma_{\mathrm{ij}}(\underline{\mathbf{n}}, \underline{\mathbf{n}})$ is the cross section for scattering of the ith mode from direction $\underline{\underline{n}}$ into the $\mathrm{jth}$ mode in direction $\underline{\mathbf{n}}^{\prime} . \sigma_{\mathbf{i}}(\underline{\underline{n}})$ is the integrated scattering coefficient - that is the extinction coefficient - for the ith mode. $\sigma_{\mathrm{i}}$ and $\sigma_{\mathrm{ij}}$ have been calculated using the expressions given by Kaminker et al (1982) which are expected to be valid away from the cyclotron resonance. $\quad \kappa_{i}^{a}(\underline{n})$ is the absorption cross section for the ith mode and has been calculated as the sum of cyclotron and free-free components following Wickramasinghe and Meggitt (1985a) 


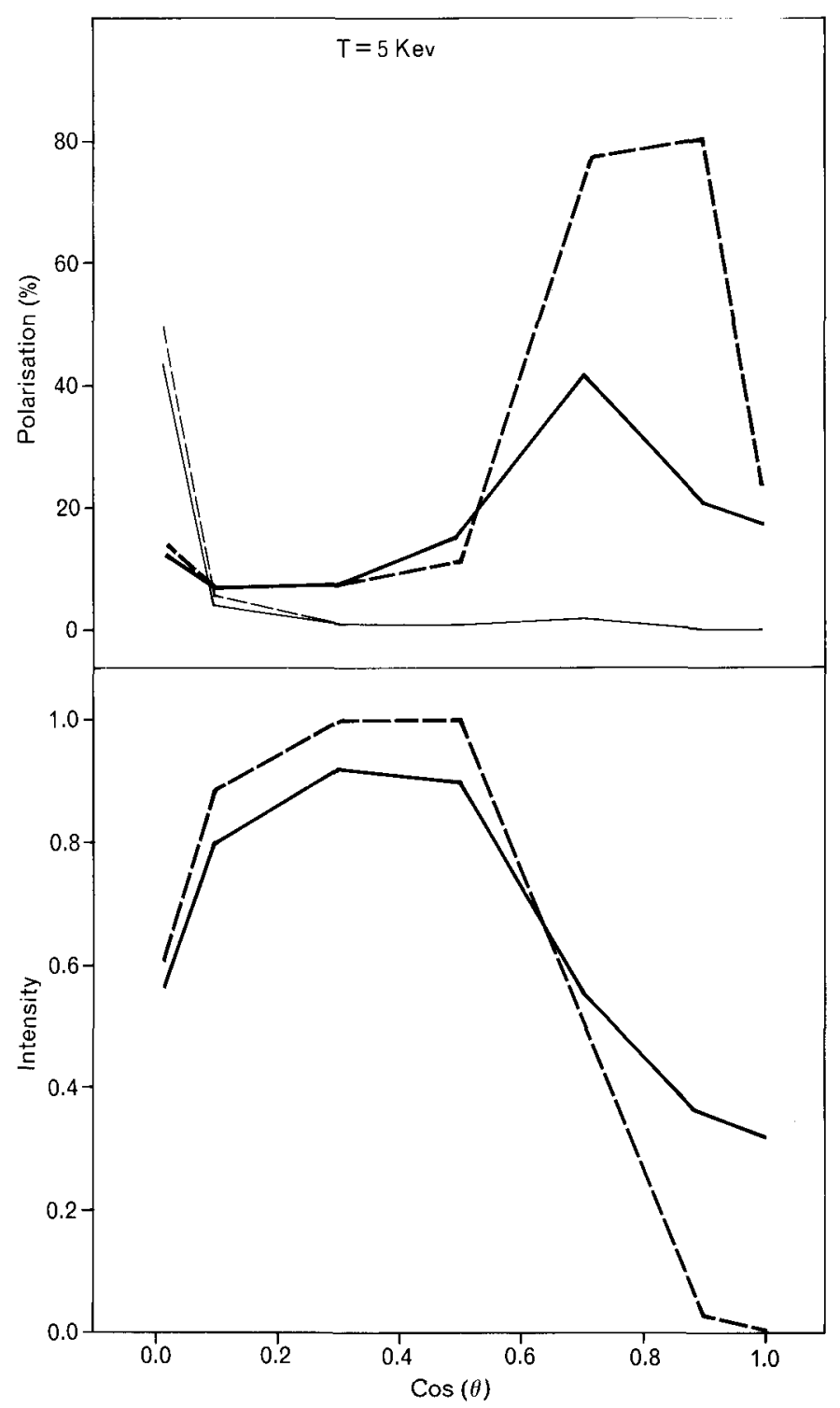

Figure 1 The properties of the radiation emerging from a cylinarical emission region with the magnetic field alligned along the cylindrical axis and with parameters as discussed in the text. The lower and upper panels present the intensity and polarisation (heavy curves-circular; light curves-linear) of the radiation at different angles $\theta$ to the magnetic field. The dashed and solid curves present calculations without and with electron scattering included in the transfer equations. 
We note that unlike in the case of pure absorption the differential equations for $I_{1}$ and $I_{2}$ are now coupled through the scattering coefficient $\sigma_{12}$. Furthermore the geometry of the emission region plays a more direct role since scattering couples the intensities in different directions.

The equations have been solved numerically using one of two methods. In the first approach the equations are discretised in angle and reformulated as an initial value problem using transmission and reflection matrices following Schmidt and Wehrse (1986). The method involves the solution of a large number of first order differential equations using a Runge-Kutta scheme. In the second approach the integro-differential equations (1) and (2) are solved by an iterative scheme (Meggitt and Wickramasinghe (1986)). The latter method was found to be more economical in computer time when the electron scattering optical depth was not too large $\left(\tau_{\mathrm{e}} \leq 5\right)$.

We present in figure 1 results oif a calculation of the emergent intensity and polarisation at various angles $\theta$ to the magnetic field for a cylindrical emission region with the magnetic field aligned along the cylindrical axis. The cylinder has a diameter $\mathrm{d}=10^{9} \mathrm{~cm}$ and a height $\mathrm{h}=10^{9} \mathrm{~cm}$. The magnetic field $\mathrm{B}=3 \times 10^{7} \mathrm{G}$, electron temperature $\mathrm{T}_{\mathrm{e}}=5 \mathrm{kev}$, electron number density $\mathrm{N}_{\mathrm{e}}=5 \times 10^{15} \mathrm{~cm}^{-3}$ and the calculations are for harmonic number $\mathbf{n}=\omega / \omega_{\mathrm{c}}=8$ where $\omega_{\mathrm{c}}$ is the cyclotron frequency. The above parameters have been chosen for illustrative purposes to highlight the effects of scattering. With these parameters the maximum absorption and scattering optical depths in directions along and perpendicular to the axis of the cylinder are $\tau_{\mathrm{e}}(\mathrm{h})=4.3$, $\tau_{\mathrm{abs}}(\mathrm{h})=0.01$ and $\tau_{\mathrm{e}}(\mathrm{d})=3.5, \tau_{\mathrm{abs}}(\mathrm{d})=20.0$ respectively indicating that the emission region is scattering dominated.

Comparing the scattered (solid curve) and unscattered (dashed curve) intensities we note that scattering has the expected effect of filling in regions of low intensity (low $\theta$ ) with scattered radiation from regions of high intensity (high $\theta$ ). However despite the large optical depths to electron scattering, the 
scattered radiation is strongly circularly polarised at low $\theta$. We note also that the linear pulse is virtually unaffected by scattering. These results should be compared with models which incorporate scattering as absorption and thermal emission terms in the transfer equations. Such models predict that at high scattering optical depths the circular polarisation would tend to zero when the emission region is viewed along the magnetic field $\left(\theta-0^{\circ}\right.$ ) (Barrett and Chanmugam (1984)).

\section{Conclusions}

Our main conclusions may be summarised as follows :

a) If the emission region is scattering dominated $\left(\tau_{\mathrm{e}}(\mathrm{h})>\tau_{\mathrm{abs}}(\mathrm{h})\right)$ the radiation at low values of $\theta$ will in general be circularly polarised $\left(\mathrm{O}\left(\omega / \omega_{\mathrm{c}}\right)\right)$ even if $\tau_{\mathrm{e}}(\mathrm{h})>>1$.

b) If the emission region is absorption dominated $\left(\tau_{\mathrm{e}}(\mathrm{h}) \ll<\tau_{\mathrm{abs}}(\mathrm{h})\right)$ the circular polarisation will tend to zero at low values of $\theta$ provided $\tau_{\mathrm{abs}}(\mathrm{h})>1$. These conditions are likely to be realised only at very low values of $\mathrm{T}_{\mathrm{e}}(<1 \mathrm{kev})$ and are unlikely to be relevant to $A M$ Herculis type systems.

c) The circular polarisation standstills characterised by zero polarisation at minimum $\theta$ observed in some AM Herculis type systems cannot be explained by cyclotron emission in a scattering dominated region as initially proposed by Barrett and Chanmugam (1984). However such standstills are readily explicable in terms of dilution by unpolarised background sources of radiation as has been demonstrated in E1405-451 (Bailey et al (1983), Wickramasinghe and Meggitt (1985b)). The electron scattering optical depth of the cyclotron emission region in such systems is likely to be $\leq 0.1$. 
d) The very low values of circular polarisation $(<1 \%)$ observed in some intermediate polars cannot be explained by cyclotron emission in a scattering dominated region unless the magnetic field strengths are $\leq 10^{6} \mathrm{G}$.

\section{References}

Bailey, J. Axon, D.J., Hough, J.H. Watts, D.J., Giles, A.B. and Greenhill, J.G., 1983, Mon. Nat. Roy. Ast. Soc. 205, 1p.

Barrett, P.E. and Chanmugam, G., 1984, Astrophys. J., 278, 298

Chanmugam, G. and Dulk, G.A., 1981, Astrophys. J., 244 , 569

Kaminker, A.D., Pavlov, G.G., and Shibanov, Yu.A., 1982, Astrophys and Space Science, 86, 249

Meggitt, S.M.A. and Wickramasinghe, D.T., 1982, Mon. Nat. R oy. Ast. Soc., 198, 71

Meggitt, S.M.A. and Wickramasinghe, D.T., 1986 (in preparation)

Schmidt, M. and Wehrse, R., 1986 in Numerical Methods in Radiative transfer (Ed. Kalkofen, W.) - Cambridge University press.

Wickramasinghe,. D.T. and Meggitt, S.M.A., 1985, Mon. Not. Roy. Ast. Soc., 214, 605

Wickramasinghe,m D.T. and Meggitt, S.M.A., 1985, Mon. Not. Roy. Ast. Soc., 216, 857 . 